\title{
ON SEMIGROUPS GENERATED BY $m$-ACCRETIVE OPERATORS IN A STRICT SENSE
}

\author{
MICHIAKI WATANABE
}

ABSTRACT. Let $\{S(t): t \geqslant 0\}$ be a nonlinear semigroup generated by an $m$-accretive operator $A$ in a real Banach space $(X,|\cdot|)$. It is shown that (1) for any $x \in \overline{D(A)}$, $S(\cdot) x$ belongs to $L^{p}(0, T ; V)(T>0)$ if $A$ satisfies

$$
\left|u_{1}-u_{2}\right|^{p}+C \lambda\left\|u_{1}-u_{2}\right\|^{p} \leqslant\left|u_{1}+\lambda A u_{1}-u_{2}-\lambda A u_{2}\right|^{p}
$$

$(p>1, C>0)$ for $\lambda>0$ and $u_{i} \in D(A)(i=1,2)$, where $(V,\|\cdot\|)$ is a Banach space including $D(A)$ and included continuously in $X$; and that (2) $A+B$ has similar properties to those of the above $A$ if $B$ is a Lipschitz continuous operator from $V$ to $X$.

1. Introduction. Let $(X,|\cdot|)$ and $(V,\|\cdot\|)$ be real Banach spaces such that $V$ is included in $X$ algebraically and continuously. Consider an operator $A$ in $X$ (possibly nonlinear and multivalued) satisfying

$$
\begin{gathered}
V \supset D(A) \quad(\text { the domain of } A) ; \\
\left|u_{1}-u_{2}\right|^{p}+C \lambda\left\|u_{1}-u_{2}\right\|^{p} \leqslant\left|u_{1}+\lambda A u_{1}-u_{2}-\lambda A u_{2}\right|^{p}
\end{gathered}
$$

for all $\lambda>0$ and $u_{i} \in D(A)(i=1,2)$, where $p>1$ and $C>0$ are constants;

$$
X=R(I+\lambda A) \quad \text { (the range of } I+\lambda A) \text { for all } \lambda>0 .
$$

Clearly conditions (B) and (C) imply that the above $A$ is $m$-accretive in $X$ and hence generates a semigroup $\{S(t): t \geqslant 0\}$ on $\overline{D(A)}$ (the closure in $X$ of $D(A)$ ). Crandall and Liggett [1] demonstrated that for each $x \in \overline{D(A)},(I+(t / n) A)^{-n} x$ converges to $S(t) x$ in $X$ as $n \rightarrow \infty$ uniformly on every bounded subinterval of $[0, \infty)$ and that $\{S(t): t \geqslant 0\}$ becomes a continuous semigroup of nonexpansive self-mappings of $\overline{D(A)}$.

The objective in this paper is to study some properties of $\{S(t): t \geqslant 0\}$ generated by an operator $A$ satisfying the conditions (A)-(C). We will show below, among other things, that for any $x \in \overline{D(A)}, S(\cdot) x$ belongs to $L^{p}(0, T ; V)$ for $T>0$, and that if an operator $B: V \rightarrow X$ is Lipschitz continuous, then $A+B$ has similar properties to those of $A$.

Received by the editors November 23, 1983 and, in revised form, September 11, 1984. This paper was presented at RIMS Kyoto University: Meeting on Nonlinear Evolution Equations and Applications, October 14, 1985.

1980 Mathematics Subject Classification. Primary 47H20, 47H06.

Key words and phrases. Semigroup, $m$-accretive operator, perturbation. 
The condition (B) is, as is easily seen, equivalent to each of the following:

$$
\tau\left(u_{1}-u_{2}, A u_{1}-A u_{2}\right)\left|u_{1}-u_{2}\right|^{p-1} \geqslant(C / p)\left\|u_{1}-u_{2}\right\|^{p},
$$

where $\tau(x, y)=\inf _{\lambda>0} \lambda^{-1}(|x+\lambda y|-|x|)$ for $x, y \in X$;

$$
\left|u_{1}-u_{2}\right|^{p}+(C / p) \lambda\left\|u_{1}-u_{2}\right\|^{p} \leqslant\left|u_{1}+\lambda A u_{1}-u_{2}-\lambda A u_{2} \| u_{1}-u_{2}\right|^{p-1}
$$

ReMarK. In the case that $X$ is a Hilbert space, condition (1.1) with $p=2$ is nothing but an inequality of Gårding type. It is also evident that the inequality

$$
\left|u_{1}-u_{2}\right|+K \lambda^{\alpha}|| u_{1}-u_{2}|| \leqslant\left|u_{1}+\lambda A u_{1}-u_{2}-\lambda A u_{2}\right|
$$

for $\lambda>0, u_{i} \in D(A)(i=1,2)$ and constants $K>0$ and $\alpha$ with $0<\alpha<1$, implies (B) with $p=\alpha^{-1}$ and $C=K^{p}$. Therefore, condition (B) is considered to be rather general.

2. Smoothness of $S(t)$. By "smoothness" we mean that for a.a. $t>0, S(t) \overline{D(A)}$ is included in $V$. The purpose of this section is to prove the following theorem.

THEOREM 1. Let $\{S(t): t \geqslant 0\}$ be the semigroup on $\overline{D(A)}$ generated by $A$ satisfying (A)-(C). Then, the following statements hold.

(i) For each $x \in \overline{D(A)}$ and $T>0, S(\cdot) x$ belongs to $L^{p}(0, T ; V)$.

(ii)

$$
|S(t) x-S(t) y|^{p}+C \int_{0}^{t}\|S(r) x-S(r) y\|^{p} d r \leqslant|x-y|^{p}
$$

for all $x, y \in \overline{D(A)}$ and $t \geqslant 0$.

(iii)

$$
\begin{gathered}
|S(t) x-v|^{p}-|x-v|^{p}+C \int_{0}^{t}\|S(r) x-v\|^{p} d r \\
\quad \leqslant p \int_{0}^{t} \tau(S(r) x-v,-A v)|S(r) x-v|^{p-1} d r
\end{gathered}
$$

for all $x \in \overline{D(A)}, v \in D(A)$ and $t \geqslant 0$.

Before giving the proof, we first prove several lemmas, of which we will make later use, under the hypothesis of the theorem.

Lemma 1. (i) For each $x \in X, J_{\lambda} x=(I+\lambda A)^{-1} x$ is continuous in $\lambda \in(0,1]$ in the topology of $V$.

(ii) For each $u \in D(A), J_{\lambda} u$ is continuous in $\lambda \in[0,1]$ in the toplogy of $V$, where $J_{0}=I$.

Proof. From (B) we see that

$$
\left\|J_{\lambda} x-J_{\lambda} y\right\| \leqslant(C \lambda)^{-1 / p}|x-y|
$$

for all $\lambda>0$ and $x, y \in X$.

Let $x \in X$ and $0<\mu<\lambda$. Then, using (2.1) and the formula

$$
J_{\lambda} x=J_{\mu}\left(\mu \lambda^{-1} x+(\lambda-\mu) \lambda^{-1} J_{\lambda} x\right),
$$


we have

$$
\begin{aligned}
\left\|J_{\lambda} x-J_{\mu} y\right\| & \leqslant(C \mu)^{-1 / p}\left|\mu \lambda^{-1} x+(\lambda-\mu) \lambda^{-1} J_{\lambda} x-x\right| \\
& =(C \mu)^{-1 / p} \cdot \lambda^{-1}(\lambda-\mu)\left|J_{\lambda} x-x\right|,
\end{aligned}
$$

which implies that (i) is true. For the proof of (ii) we have only to show the continuity at $\lambda=0$ of $J_{\lambda} u$ for $u \in D(A)$. Again using (2.1), we obtain

$$
\left\|J_{\lambda} u-u\right\| \leqslant(C \lambda)^{-1 / p}|u-(I+\lambda A) u|=C^{-1 / p} \cdot \lambda^{1-1 / p}|A u| \text {. Q.E.D. }
$$

Lemma 2. Let $\{S(t): t \geqslant 0\}$ be the semigroup generated in $X$ by $A$. Then, for each $u \in D(A)$ and $t \geqslant 0, S(t) u$ belongs to $V$, and the following statements hold.

(i) $\left\|J_{t / n}^{n} u-S(t) u\right\| \rightarrow 0$ as $n \rightarrow \infty$ uniformly on every bounded subinterval of $[0, \infty)$.

(ii) $S(t) u$ is continuous in $t \geqslant 0$ in the topology of $V$ and satisfies, for all $t, s \geqslant 0$,

$$
\|S(t) u-S(s) u\| \leqslant 2(p / C)^{1 / p}|A u \| t-s|^{1-1 / p} .
$$

Proof. By (1.1) we have

$$
\left\|J_{\lambda}^{m} u-J_{\mu}^{n} u\right\|^{p} \leqslant(p / C)\left(\left|A J_{\lambda}^{m} u\right|+\left|A J_{\mu}^{n} u\right|\right)\left|J_{\lambda}^{m} u-J_{\mu}^{n} u\right|^{p-1} .
$$

Since $\left|A J_{\lambda}^{n} u\right| \leqslant|A u|$, this estimate becomes

$$
\left\|J_{\lambda}^{m} u-J_{\mu}^{n} u\right\| \leqslant(2 p / C)^{1 / p}|A u|^{1 / p}\left|J_{\lambda}^{m} u-J_{\mu}^{n} u\right|^{1-1 / p} .
$$

Using the estimate due to Crandall and Liggett [1, (1.9)], we obtain

$$
\begin{aligned}
\left\|J_{\lambda}^{m} u-J_{\mu}^{n} u\right\| \leqslant(2 p / C)^{1 / p}|A u| & \left\{(m \lambda-n \mu)^{2}+n \mu(\lambda-\mu)\right\}^{1 / 2} \\
& \left.+\left\{(m \lambda-n \mu)^{2}+m \lambda(\lambda-\mu)\right\}^{1 / 2}\right]^{1-1 / p}
\end{aligned}
$$

if $0<\mu \leqslant \lambda$ and $0<m \leqslant n$ (integers).

Putting $\mu=t / n$ and $\lambda=t / m$ yields

$$
\left\|J_{t / m}^{m} u-J_{t / n}^{n} u\right\| \leqslant 2(p / C)^{1 / p}|A u|\left\{t(1 / m+1 / n)^{1 / 2}\right\}^{1-1 / p},
$$

which implies that $J_{t / n}^{n} u$ converges in $V$ to some $v(t)$ uniformly on every bounded subinterval of $[0, \infty)$. But, since $V \subset X$ continuously, $v(t)$ coincides with $S(t) u$ for all $t \geqslant 0$.

The assertion (ii) is proved by taking the limit in (2.2) with $m=n, \mu=t / n$ and $\lambda=s / m$. Q.E.D.

LemMa 3. For all $u, v \in D(A)$ and $t \geqslant 0$

$$
|S(t) u-S(t) v|^{p}+C \int_{0}^{t}\|S(r) u-S(r) v\|^{p} d r \leqslant|u-v|^{p} .
$$

Proof. Using (B), we have

$$
C \lambda\left\|J_{\lambda}^{i} x-J_{\lambda}^{i} y\right\|^{p} \leqslant\left|J_{\lambda}^{i-1} x-J_{\lambda}^{i-1} y\right|^{p}-\left|J_{\lambda}^{i} x-J_{\lambda}^{i} y\right|^{p}
$$

for $x, y \in X$ and $i=1,2, \ldots, n$. 
Summing up the above for $i=1,2, \ldots, n$, we obtain

$$
\left|J_{\lambda}^{n} x-J_{\lambda}^{n} y\right|^{p}+C \sum_{i=1}^{n} \lambda\left\|J_{\lambda}^{i} x-J_{\lambda}^{i} y\right\|^{p} \leqslant|x-y|^{p},
$$

which implies, by Lemma 1 , that for all $u, v \in D(A)$ and $\lambda>0$

$$
\left|J_{\lambda}^{n} u-J_{\lambda}^{n} v\right|^{p}+C \int_{\lambda}^{(n+1) \lambda}\left\|J_{\lambda}^{[r / \lambda]} u-J_{\lambda}^{[r / \lambda]} v\right\|^{p} d r \leqslant|u-v|^{p} .
$$

Noting Lemma 2 and taking the limit as $\lambda \downarrow 0$ with $t \geqslant \lambda$ and $n=[t / \lambda]$, we obtain (2.3). Q.E.D.

LEMMA 4. For all $u, v \in D(A)$ and $t \geqslant 0$

$$
\begin{aligned}
& |S(t) u-v|^{p}-|u-v|^{p}+C \int_{0}^{t}\|S(r) u-v\|^{p} d r \\
& \quad \leqslant p \int_{0}^{t} \tau(S(r) u-v,-A v)|S(r) u-v|^{p-1} d r .
\end{aligned}
$$

Proof. Put $x_{i}=J_{\lambda}^{i} u$. Then, using (1.1) and recalling the properties of $\tau(\cdot, \cdot)$, we have

$$
\begin{aligned}
(C / p)\left\|x_{i}-v\right\|^{p} \leqslant & \tau\left(x_{i}-v, A x_{i}-A v\right)\left|x_{i}-v\right|^{p-1} \\
= & \tau\left(x_{i}-v, \lambda^{-1}\left(x_{i-1}-x_{i}\right)-A v\right)\left|x_{i}-v\right|^{p-1} \\
\leqslant & -\lambda^{-1}\left|x_{i}-v\right|^{p}+\lambda^{-1}\left|x_{i-1}-v\right|\left|x_{i}-v\right|^{p-1} \\
& +\tau\left(x_{i}-v,-A v\right)\left|x_{i}-v\right|^{p-1} \\
\leqslant & (p \lambda)^{-1}\left|x_{i-1}-v\right|^{p}-(p \lambda)^{-1}\left|x_{i}-v\right|^{p} \\
& +\tau\left(x_{i}-v,-A v\right)\left|x_{i}-v\right|^{p-1}
\end{aligned}
$$

Consequently we obtain

$$
\left|x_{i}-v\right|^{p}-\left|x_{i-1}-v\right|^{p}+C \lambda\left\|x_{i}-v\right\|^{p} \leqslant p \lambda \cdot \tau\left(x_{i}-v,-A v\right)\left|x_{i}-v\right|^{p-1} .
$$

Summing up the above for $i=1,2, \ldots, n$, we have

$$
\begin{gathered}
\left|x_{n}-v\right|^{p}-|u-v|^{p}+C \sum_{i=1}^{n} \lambda\left\|x_{i}-v\right\|^{p} \\
\leqslant p \sum_{i=1}^{n} \lambda \tau\left(x_{i}-v,-A v\right)\left|x_{i}-v\right|^{p-1}
\end{gathered}
$$

and hence

$$
\begin{aligned}
& \left|J_{\lambda}^{n} u-v\right|^{p}-|u-v|^{p}+C \int_{\lambda}^{(n+1) \lambda}\left\|J_{\lambda}^{[r / \lambda]} u-v\right\|^{p} d r \\
& \quad \leqslant p \int_{\lambda}^{(n+1) \lambda} \tau\left(J_{\lambda}^{[r / \lambda]} u-v,-A v\right)\left|J_{\lambda}^{[r / \lambda]} u-v\right|^{p-1} d r .
\end{aligned}
$$

Since $\tau(\cdot, \cdot)$ is upper semicontinuous and the integrand of the right is dominated by $p|A v|(r|A u|+|u-v|)^{p-1}$, going to the limit as $\lambda \downarrow 0$ with $t \geqslant \lambda$ and $n=[t / \lambda]$, we obtain (2.4) (see [3, pp. 249-250]). Q.E.D. 
Proof of TheOREM 1. Take $x \in \overline{D(A)}$ and let $\left\{u_{n}\right\}_{n=1}^{\infty}$ be a sequence in $D(A)$ such that $\left|u_{n}-x\right| \rightarrow 0$ as $n \rightarrow \infty$. Using (2.3), we have, for $T>0$,

$$
\left|S(T) u_{m}-S(T) u_{n}\right|^{p}+C \int_{0}^{T}\left\|S(r) u_{m}-S(r) u_{n}\right\|^{p} d r \leqslant\left|u_{m}-u_{n}\right|^{p},
$$

which implies that $S(\cdot) u_{n}$ converges in the topology of the Banach space $L^{p}(0, T ; V)$ to some $w$ as $n \rightarrow \infty$.

But, $V$ is included in $X$ continuously and $S(\cdot) u_{n}$ tends to $S(\cdot) x$ in $L^{p}(0, T ; X)$ as $n \rightarrow \infty$. Hence, $w(t)$ must coincide with $S(t) x$ for a.a. $t \in(0, T)$, which completes the proof of (i).

The proof of (ii) and (iii) is simple. Let $x, y \in \overline{D(A)}$ and $\left\{u_{n}\right\}_{n=1}^{\infty}$ and $\left\{v_{n}\right\}_{n=1}^{\infty}$ be sequences in $D(A)$ such that $\left|u_{n}-x\right| \rightarrow 0$ and $\left|v_{n}-y\right| \rightarrow 0$ as $n \rightarrow \infty$. We have only to replace $u, v$ in (2.3) and $u$ in (2.4) by $u_{n}, v_{n}$ and $u_{n}$ respectively, and only to take the limit as $n \rightarrow \infty$. Q.E.D.

3. Perturbation for $S(t)$. Let $A$ be an operator satisfying (A)-(C). The purpose of this section is to give conditions on an operator $B$ in $X$ under which $A+B$ also satisfies such conditions as (A)-(C).

We impose on $B$ the following:

$$
V \subset D(B) ;
$$

$$
\begin{aligned}
& \text { There is a constant } L \geqslant 1 \text { such that }|B u-B v| \leqslant L\|u-v\| \\
& \text { for all } u, v \in V .
\end{aligned}
$$

The Lipschitz continuity condition of $B: V \rightarrow X$ is simple but new. In fact, it is possible for $B$ to stand for a "differential" operator. In perturbation theory in this direction for nonlinear semigroups, $A$ was assumed to be $m$-accretive simply but $B$ was sometimes forced to satisfy the condition $\overline{D(A)} \subset D(B)$ instead (see e.g. $[4,6]$ ), which is too restrictive for $B$ to become a "differential" operator.

Our method can be used to treat the same problem in the case that $B$ satisfies a "local" Lipschitz continuity condition from $V$ to $X$. In this paper, however, to clarify our idea we confine ourselves to conditions (D) and (E).

Theorem 2. If $A$ satisfies (A)-(C) and B satisfies (D) and (E), then $A+B$ has the following properties:

$$
\begin{gathered}
V \supset D(A+B) \\
(1-\omega \lambda)\left|u_{1}-u_{2}\right|^{p}+(C /(2 p)) \lambda\left\|u_{1}-u_{2}\right\|^{p} \\
\leqslant\left|u_{1}-\lambda(A+B) u_{1}-u_{2}-\lambda(A+B) u_{2}\right|\left|u_{1}-u_{2}\right|^{p-1}
\end{gathered}
$$

for all $\lambda>0$ and $u_{i} \in D(A+B)(i=1,2)$;

$$
R(I+\lambda(A+B))=X \text { for all } \lambda \text { with } 0<\lambda<\omega^{-1},
$$

where $\omega=(1-1 / p) L^{p /(p-1)}(2 / C)^{1 /(p-1)}$.

Proof. Since $D(A+B)=D(A)$, (a) is obvious. 
Using (1.2) and (E), we have for $\lambda>0$ and $u_{i} \in D(A)(i=1,2)$

$$
\begin{aligned}
& \left|u_{1}-u_{2}\right|^{p}+(C / p) \lambda\left\|u_{1}-u_{2}\right\|^{p} \\
& \quad \leqslant\left(\left|u_{1}+\lambda(A+B) u_{1}-u_{2}-\lambda(A+B) u_{2}\right|+L \lambda\left\|u_{1}-u_{2}\right\|\right)\left|u_{1}-u_{2}\right|^{p-1} .
\end{aligned}
$$

Applying to the right the inequality

$$
L \cdot Y Z^{p-1} \leqslant(C /(2 p)) Y^{p}+\omega Z^{p} \text { for nonnegative } Y \text { and } Z,
$$

which is a simple consequence of Young's inequality, we see that (3.1) implies (b).

To prove (c) we will show that for each $x \in X$ there exists $u \in D(A)$ such that $u+\lambda(A+B) u=x$. Put $T u=(I+\lambda A)^{-1}(x-\lambda B u)$ for $\lambda>0$. Then the operator $T$ maps $V$ into $D(A)$ and hence into $V$. In view of (1.2) we see that $T$ satisfies

$$
\begin{aligned}
\left|T u_{1}-T u_{2}\right|^{p}+(C / p) \lambda\left\|T u_{1}-T u_{2}\right\|^{p} & \leqslant \lambda\left|B u_{1}-B u_{2}\right|\left|T u_{1}-T u_{2}\right|^{p-1} \\
& \leqslant L \lambda\left\|u_{1}-u_{2}\right\|\left|T u_{1}-T u_{2}\right|^{p-1}
\end{aligned}
$$

for all $\lambda>0$ and $u_{i} \in V(i=1,2)$.

Again using (3.2), we have

$$
(1-\omega \lambda)\left|T u_{1}-T u_{2}\right|^{p}+(C / p) \lambda\left\|T u_{1}-T u_{2}\right\|^{p} \leqslant(C /(2 p)) \lambda\left\|u_{1}-u_{2}\right\|^{p} .
$$

Hence, if $0<\lambda<\omega^{-1},\left\|T u_{1}-T u_{2}\right\| \leqslant 2^{-1 / p}\left\|u_{1}-u_{2}\right\|$. Since $2^{-1 / p}$ is smaller than 1 , a well-known fixed point theorem applies. Let $u$ be the unique fixed point of $T$ in $V$. Then $u=(I+\lambda A)^{-1}(x-\lambda B u)$, which implies that $u$ belongs to $D(A)$ and satisfies $u+\lambda(A+B) u=x$. Q.E.D.

EXAmple. Let $\Omega$ be a bounded domain in $R^{N}$ with smooth boundary. Consider the natural realization $A$ in $L^{2}(\Omega)$ of $-\Delta, \Delta=\sum_{i=1}^{N}\left(\partial / \partial x_{i}\right)^{2}$ with $D(A)=H^{2}(\Omega)$ $\cap H_{0}^{1}(\Omega)$, where $H^{k}(\Omega)$ and $H_{0}^{k}(\Omega)$ denote the usual Sobolev spaces-the real Hilbert spaces with norm $\|\cdot\|_{k}$ and inner product $(\cdot, \cdot)_{k}$. Integration by parts yields $(A u, u)_{0}=\|u\|_{1}^{2}-\|u\|_{0}^{2}$ for $u \in D(A)$, a special form of Garding's inequality. Hence, the linear operator $A$ satisfies the condition

$$
(1-\lambda)\|u\|_{0}^{2}+\lambda\|u\|_{1}^{2} \leqslant\|u+\lambda A u\|_{0}\|u\|_{0}
$$

as well as $R(I+\lambda A)=L^{2}(\Omega)$ for $0<\lambda<1$ (Lax-Milgram Lemma). Next, the operator $B$ defined on $H_{0}^{1}(\Omega)$ by $B u=\left(1+|\operatorname{grad} u|^{2}\right)^{1 / 2}$ clearly satisfies

$$
\|B u-B v\|_{0} \leqslant\|u-v\|_{1} \text { for } u, v \in H_{0}^{1}(\Omega) .
$$

Thus, we can conclude by Theorems 1 and 2 that for the nonlinear semigroup $\{T(t): t \geqslant 0\}$ in $L^{2}(\Omega)$ generated by the semilinear operator $A+B, T(t) L^{2}(\Omega)$ is included in $H_{0}^{1}(\Omega)$ for a.a. $t>0$.

REMARK. It is known $[2,5]$ that if $X$ is a "nice" Banach space, then there is a biunique correspondence between $m$-accretive operators in $X$ and nonlinear semigroups on nonexpansive retracts of $X$. It would be of interest to determine if there is an analogous correspondence between $m$-accretive operators satisfying (B) and nonlinear semigroups which satisfy (ii) of Theorem 1 . 
The author wishes to thank the referee for useful suggestions.

NoTE ADDED IN PROOF. Since this paper was submitted for publication, we have become aware that the theory in $\$ 2$ resembles that in $\S 3$ of the paper:

A. Pazy, The Lyapunov method for semigroups of nonlinear contractions in Banach spaces, J. Analyse Math. 40 (1981), 239-262.

Here, the semigroup generated by $A$ which satisfies instead of (B)

$$
\phi(u)+\lambda \psi(u) \leqslant \phi(u+\lambda A u) \text { for } u \in D(A) \text { and } \lambda>0,
$$

where $\phi$ and $\psi$ are lower semicontinuous functions, roughly speaking, is discussed among other things.

The author is grateful to Professor A. Pazy for his kind advice concerning this work.

\section{REFERENCES}

1. M. G. Crandall and T. M. Liggett, Generation of semigroups of nonlinear transformations on general Banach spaces, Amer. J. Math. 93 (1971), 265-298.

2. M. G. Crandall and A. Pazy, Semigroups of nonlinear contractions and dissipative sets, J. Funct. Anal. 3 (1969), 376-418.

3. I. Miyadera, Some remarks on semigroups of nonlinear operators, Tôhoku Math. J. 23 (1971), 245-258.

4. M. Piérre, Perturbations localement Lipschitziennes et continues d'opérateurs m-accrétifs, Proc. Amer. Math. Soc. 58 (1976), 124-128.

5. S. Reich, Product formulas, nonlinear semigroups, and accretive operators, J. Funct. Anal. 36 (1980), 147-168.

6. G. Webb, Continuous nonlinear perturbations of linear accretive operators in Banach spaces, J. Funct. Anal. 10 (1972), 191-203.

Faculty of General Education, Nilgata University, IKarashi 2 - 8050, Niigata, Japan 950 - 21 